\title{
Effects of particle elongation on the binary coalescence dynamics of powder grains for Laser Sintering applications
}

\author{
S. Haeri ${ }^{\mathrm{a}, *}$ L. Benedetti ${ }^{1}$, O. Ghita ${ }^{1}$ \\ ${ }^{a}$ James Weir Fluid Laboratory, Department of Mechanical and Aerospace Engineering, \\ University of Strathclyde, Glasgow G1 1XJ, UK \\ ${ }^{b}$ College of Engineering, Mathematics and Physical Sciences, University of Exeter, North \\ Park Road EX4 4QF, UK
}

\begin{abstract}
In this paper, the effects of particle shape and viscoelasticity on the binary coalescence rates of a pair of PEK HP3 particles in the Laser Sintering (LS) process are investigated in detail. PEK HP3 powder was characterised and the sintering rates were determined using Hot Stage Microscopy (HSM). On average, the neck growth rate shows a slower dynamic compared to the theory. Furthermore, in some of the trials an initial delay in growth and/or a contraction in the neck size was observed. To understand this deviation, the dynamics of individual non-spherical particles were studied at similar conditions. The overall perimeter of the particles' shrank and they gradually attained a final spherical state under the effect of surface tension forces which indicates that shape of the individual coalescing units can influence the sintering rates. In addition, the effects of viscoelasticity were investigated using the available theoretical approaches and results show that viscoelasticity can change the rate of coalescence but the neck growth rate shows a strictly increasing trend even for very large Deborah numbers. To
\end{abstract}

\footnotetext{
${ }^{*}$ Corresponding author.

Email address: sina.haeri@strath.ac.uk (S. Haeri)
} 
investigate the shape effects, volume-of-fluid ( VoF) simulations of elongated ellipsoidal particles in head-to-head contact were performed and the neck growth rates were determined. These analyses show that the contraction of the neck size can occur purely due to the geometrical effects and local surface curvatures of the coalescing particles. In addition, a critical aspect ratio exists beyond which the coalescence does not complete and the contraction continues until the pair of coalescing particles eventually separate and form two independent spheres. To explain this phenomenon, the local curvatures along the surface of coalescing particles were calculated and the results show that a local minimum in the curvature at the neck's contact points with the particle's main body forms which is believed to drives the separation.

Keywords: Laser Sintering; Powder Bed Fusion; Additive Manufacturing; Non-spherical Particles; Volume of Fluid.

\section{Introduction}

Powder bed fusion is a promising Additive Manufacturing (AM) technology where polymeric (or metallic) powders are spread on a fabrication piston to form a thin (typically less than $0.1 \mathrm{~mm}$ in thickness) bed of powder using a counter-rotating roller or a blade. Different technologies may then be used to fuse the powder (see Figure 1). For example, in a Laser Sintering (LS) process, a laser beam is used to fuse the grains. After this stage, the fabrication piston lowers the part slightly and a new layer of powder is applied. The process is repeated until the production is completed [1].

A successful fabrication depends on an appropriate choice of the process parameters which should be decided based on the powder characteristics and its dynamical response to the various stages of the process. Particle 
shape and size have a great impact on the powder spreading (re-coating) process and the characteristics of the powder bed such as the solid volume fraction [1-3]. After the spreading, particles are melted using a laser beam to bind them through a sintering process. Generally, sintering occurs either at a solid state due to molecular/atomic diffusion at temperatures below the melting point or happens in a liquid phase at temperatures higher than the melting point. For semi-crystalline polymers near and above the melting point (conditions similar to the LS process), a viscous flow induced by the surface tension forces is the dominant sintering mechanism. Throughout this paper, we are only concerned with viscous sintering and hence, we simply refer to it as sintering or coalescence which are used interchangeably. Furthermore, during the LS process powder grains undergo a phase change. However, for consistency, we refer to the powder grains simply as "particles" regardless of whether they are in a solid or liquid phase.

During the initial stage of sintering a liquid neck forms and starts to grow between particles. The neck growth rate is a pivotal parameter in LS to make informed decisions about the process parameters such as the laser scan speed to optimise the process and hence directly affects the quality of the final part. Analytical models are available for sintering of two spherical particles. Frenkel [4] derived an equation for initial stage sintering of two spherical particles by balancing the work of surface tension, due to the decreasing surface area with the energy dissipation due to a viscous flow. Frenkel used an asymptotic approximation to describe the initial stage of the process and showed the neck grows as $R_{n}(t) / R_{p}(0) \propto t^{* 1 / 2}$, where $R_{n}$ and $R_{p}(0)$ are the neck and initial particles' radii. Furthermore, $t^{*}$ is the non-dimensional time which is defined as $t^{*}=t \sigma /\left[R_{p}(0) \eta\right]$, where $\sigma$ and $\eta$ are the surface tension coefficient and viscosity. Frenkel s theory was later corrected by Eshelby [5] 
to satisfy the continuity equation.

Frenkel]s theory cannot be applied to late-stage sintering of two spheres since the variation of particles' radii with time is not considered. Pokluda et al. 6] used conservation of mass assumption to write an equation relating $R_{p}(t)$ to the initial radius and derived a generalised model to be applied to the early as well as the late stage sintering. Pokluda et al. [6] model is later extended to include the viscoelasticity effects of polymeric liquids by Bellehumeur et al. [7]. They used the convected Maxwell model [8] to consider the viscoelasticity of the polymeric liquid and assumed a purely extensional quasi-steady state flow to derive an equation for the rate of neck growth $R_{n}(t)$. Scribben et al. 9] provided a minor improvement to Pokluda's model [6] for late stage sintering and also modified Bellehumeur's model [7] to handle general viscoelastic constitutive models.

A geometric model for binary sintering of unequal spherical particles is developed by Xie [10]. Eggersdorfer et al. [11] also proposed an analytical model to predict viscous sintering of amorphous polymers with spherical particles of arbitrary size as the basic sintering unit. Furthermore, 2D sintering models for disks (or two infinite cylinder) are also available in the literature [12, 13]. However, the shape effects have not been included in these analytical models.

Numerical simulations were also used to determine the rate of neck growth between two coalescing particles. Early Finite Element (FE) simulations of the phenomenon were limited to binary sintering of equal-sized spherical particles [14, 15]. Later, coalescence of heterogeneous spherical particles was considered by Yadha and Helble [16] using analytical and also a Boundary Integral approach. The coalescence of spherical nanoparticles was investigated using Molecular Dynamics (MD) simulations by a number 
of authors [17, 18] to determine the temperature effects. Kirchhof et al. [19] considered a sphere as the fundamental unit of sintering and simulated the sintering of agglomerate chains with different structures. Very recently, Balemans et al. [20] modelled the binary sintering of spherical particles by considering the temperature effect in conditions relevant to LS. Moreover, Kamyabi et al. 21] have provided a critical review of literature on sintering of amorphous powders.

The reviewed literature all assumed the fundamental unit of sintering to be a sphere and shape effects on the neck growth rates were not considered. The particle shape introduces two significant difficulties into the analysis since the fundamental unit is not geometrically symmetric any more. Firstly, a shape parameter should be introduced into the analysis and secondly, the contact orientation becomes relevant. In this paper, experimental, analytical and numerical techniques are used to elucidate the complex neck-growth behaviour of elongated PEK HP3 powder grains in relation to the LS process. In this paper only elongated particles are considered which can be parametrised by an aspect ration $\mathrm{A}_{r}$.

In this paper firstly, PEK HP3 particle characterisation is performed and then neck growth rates of PEK HP3 particles in binary sintering are calculated using Hot-Stage Microscopy (HSM) at the maximum heating rate to simulate the LS process. A novel approach is then used by measuring the perimeter of individual particles to investigate their evolution in time and how this affects the coalescence. It is postulated that the grain shape and viscoelasticity have a pivotal role in determining the behaviour of coalescing particles. The impact of viscoelasticity on the sintering of PEK powder (assuming spherical grain shapes) is analysed analytically and is discussed in relation to the experimental measurements. To investigate the shape 
effects, high-fidelity simulations of coalescing ellipsoidal particles in headto-head contact is performed with different aspect-ratios using a Volume of Fluid (VoF) approach and the rate of neck growth is reported as a function of aspect ratio.

\section{Experimental Methods and Results}

\subsection{Material}

The experiments were conducted with PEK HP3 for laser sintering application supplied by EOS E-Manufacturing Solutions [22]. Thermal analysis results of HP3 PEK compared with conventional PEEK are available in [23]. This confirms that HP3 is indeed PEK and not PEEK as advertised by the company, and hence we use PEK HP3 to refer to this grade of material in this paper. The viscosity, glass transition temperature $\left(T_{g}\right)$ and melting temperature $\left(\mathrm{T}_{\mathrm{m}}\right)$ of this grade are listed in Table 1

The PSD was determined using a Malvern Mastersizer 3000E laser diffraction equipment. The sample was dispersed at room temperature in a liquid solution of $0.4 \%$ of sodium hexametaphosphate to prevent the stabilization of bubbles while measuring particle size. The test was repeated three times, with five measurements collected at each repeat. The particle size distribution of PEK HP3 is shown in Figure 2. This grade presents a $D_{50}$ of $49 \mu \mathrm{m}$.

\subsection{Analysis of Neck Growth Rates}

\subsubsection{Methods}

Hot Stage Microscopy (HSM) was performed to assess particle behaviour and neck growth of PEK HP3 particles. The test used a Linkam THMS600 
microscope stage coupled with a Bruker IRScope II and connected to a DinoEye eyepiece camera. The IRScope operated in reflection mode and in the range of the visual spectrum, at a magnification of 400 times. The DynoEye model is AM423X and the camera has a resolution of 1.3 megapixel. To simulate the Laser Sintering process as well as possible, the test was performed dynamically at a heating rate of $120^{\circ} \mathrm{C} \cdot \mathrm{min}^{-1}$ from room temperature to $450^{\circ} \mathrm{C}$ which is equivalent to a non-dimensional time $t_{\mathrm{end}}^{*}$. The heating rate is the maximum rate allowed by the hot stage platform, and is believed to be much better compared to the standard heating rate selected in previous works [24, 25] to relate coalescence in hot stage to the LS process.

The pictures were captured every $2 \mathrm{~s}$, hence in an interval of $4^{\circ} \mathrm{C}$ per picture, with the assistance of DinoCapture 2.0 software. The particles were placed on glass slides, following most of the experimental studies with HSM in literature [24, 26, 27]. The images were treated and analysed using ImageJ software. The analysis consisted of the following stages:

1. The scale bars are converted into pixels;

2. The particles in analysis are manually contoured before image treatment;

3. The image is converted to binary;

4. The threshold which best describes the original particle is applied;

5. Final particle dimensions and neck are measured.

The contouring was crucial to prevent image blur affecting the boundaries of each particle, therefore leading to a more accurate description of particle change and neck growth. The temperature at which neck is formed was selected as the starting point followed by 10 subsequent pictures captured in an interval of 20 seconds between neck formation and the end of the 
analysis. The analysis of individual particles used perimeter and aspect ratio values, and neck growth used perimeter values following the methodology described in [26].

\subsubsection{Results and Discussions}

For assessment of the neck growth rate, a total of seven pairs of particles in contact with each other were selected and images similar to Figure 3 were obtained for each case. The images are then processed to calculate the neck size $R_{n}$. Figure 4 summarizes the results achieved for when two particles adjacent to each other are subjected to heating. All the curves in Figure 4 are non-dimensionalised using an averaged equivalent diameter defined as $R_{\text {eqv }}(0)=(14 \pi)^{-1} \sum_{n=1}^{7} P_{n}(0)=33.95 \mu \mathrm{m}$, where $P_{n}(0)$ is the initial perimeter of particle $\mathrm{n}(1 \leq n \leq 7)$. This is equivalent to the neck growth assessment procedure "N3" which is proposed in [26] and is found to significantly reduce the standard deviation in the experimental measurements.

For PEK HP3, sintering occurs at around $T_{f} \approx T_{m}=386^{\circ} \mathrm{C}$ (this is equivalent to $t^{*}=0$ ) and no substantial difference is visible in the length of contact between the particles before then. High frequency pictures are captured - at $0.5 s^{-1}$, see Section 2.2.1 - from $T_{f}$ to $T_{f}+24^{\circ} \mathrm{C}$ which is equivalent to $t_{\max }^{*}=130.2$ (non-dimensionalised using $R_{\text {eqv }}(0)=33.95 \mu \mathrm{m}$ as the length scale). Overall, the neck achieved approximately $80 \%$ of the equivalent diameter of the particles. This seems to be mostly an effect of heating applied to the system rather than flattening of the particles on the glass surface. This is supported by [26], which reports that neck growth of PAEKs is not significantly affected by the contact of the glass surface with the pair of particles in analysis. This was experimentally assessed by 
comparing neck growth of a pair of particles on glass blades and on glass coated with silicone based separator spray which prevents wettability.

The theoretical neck growth rate calculated from the modified Pokluda' s theory [9] (see Section 3), is also presented in Figure 4. Two major differences between the experimental results and the theory can be identified. Firstly, the overall rate of coalescence is much slower compared to the theory. Secondly, during the initial stage of sintering in some case a delay in growth and/or a contraction in the neck size in observed (e.g. see Trial No. 2, 3, 6 and 7) which manifests itself in a very slow growth rate of the averaged curve between $t^{*}=0$ and $t^{*} \approx 20$.

However, PEK HP3 approaches a spherical shape with the increase in temperature as expected from the theory [4, 6] which is demonstrated in Figure 5. In this figure, three images of particles coalescence were overlapped at different stages to compare the change in morphology, size and the coalescence progress. Similar results were achieved with the coalescence of the other particles under evaluation. In this figure a change in morphology of the individual grains during the neck growth period is also visible.

The available theoretical results based on Frenkel's model assume spherical coalescing units and hence the morphological changes only occur at the neck region. Frenkel's model does not consider the possible morphological changes that can occur on the surface of particle away from the contact point. However, during the coalescence of non-spherical particles there are morphological evolutions both at the neck region and also on the surface of individual units.

To show the tendency of individual particles to become more spherical independent of the presence of a neck, single particle experiments are performed. A total of ten particles were tracked with the HSM from room tem- 
perature to $T_{f}+24^{\circ} \mathrm{C}$ which corresponds to $t_{\max }^{*}=151.14$. Also note that the length scale used to calculate $t^{*}$ is the average radius of the set which is defined by $R_{\text {eqv }}(0)=(20 \pi)^{-1} \sum_{n=1}^{10} P_{n}(0)=29.25 \mu \mathrm{m}$ and is different from $R_{e q v}(0)$ of the set of particles used for the neck growth measurements. Figure 6 clearly demonstrates the evolution of a typical particle which attains a final spherical shape. Figure $7 \mathrm{a}$ summarizes the results achieved for the evolution of particle's perimeter when subjected to heating and the changes in the aspect ratios are presented in Figure $7 \mathrm{~b}$.

The change in perimeter suggest a shrinkage prior and during the melting for PEK HP3 starting at $t^{*} \approx 45$ which is usually followed by particle growth from $t^{*} \approx 90$. The reduction in average perimeter in Figure $7 \mathrm{a}$ is expectable due to surface tension acting to minimise the particles' surface area (e.g. by flattening of the roughness elements on the surface). Concerning the expansion, PEK HP3 particles have their average perimeter increased by approximately $8 \%$ starting at $t^{*} \approx 90$ until the end of experiments at $t_{\max }^{*}=151.14$. This expansion is possibly a result of porosity loss and thermal expansion. Nonetheless, the final perimeter is reduced by around $9 \%$ compared to its value at $t^{*}=0$. Furthermore, Figure $7 \mathrm{~b}$ shows a strictly decreasing trend in aspect ratio as a result of particles evolution to attain a more spherical shape. To better compare the change in size and morphology, the images in Figure 6 were overlapped and the result is presented in Figure 8; similar results were achieved for the other individual particles under analysis which further confirm the change in morphology at the level of individual grains involved in the sintering. 


\subsubsection{Discrepancies between the experiments and Frenkel's model}

The observed neck growth rates are quite complex and counter-intuitive. However, considering Figures 5 and 8 it is clear that the surface tension acts at the single particle level as well as on the neck. Therefore, unless the individual particles are fully spherical the surface tension forces will act to change the morphology of the entire surface rather than just the neck region. Furthermore, viscoelastic behaviour of the polymer melt is believed to contribute to this complex behaviour.

To systematically investigate these effects, the coalescence of two viscoelastic spherical particles are considered analytically, and then numerical simulation of the coalescence of two elongated particles are performed (assuming a Newtonian rheology). These studies are performed at idealised conditions compared to the experiments. This allows us to isolate the shape and viscoelastic effects and to investigate them independently.

\section{Effects of viscoelasticity}

To demonstrate the viscoelasticity effects on the neck growth rate of PEK HP3 particles, the sintering model of Bellehumeur [7] is used. A schematic presentation of the neck evolution and the relevant geometrical parameters is provided in Figure 9a. The model results in an implicit ODE for the rate of growth of sintering angle $\theta(t)$ as follows [7]:

$$
8\left(\lambda K_{1} \frac{d \theta}{d t}\right)^{2}+\left(-2 \lambda K_{1}+\frac{\eta R_{p}(0)}{\sigma} \frac{K_{1}^{2}}{K_{2}}\right) \frac{d \theta}{d t}-1=0,
$$

where, $\lambda, \sigma, \eta$ and $\theta$ are the relaxation time, surface tension, viscosity and sintering angle. The sintering angle is related to the neck and particle radii 
by [6]:

$$
R_{n}(t)=R_{p}(t) \sin (\theta(t))
$$

where, $R_{p}(t)$ is given by [6]:

$$
R_{p}(t)=R_{p}(0)\left(\frac{4}{[1+\cos (\theta(t))]^{2}[2-\cos (\theta(t))]}\right)^{1 / 3} .
$$

In Eq. (1), $K_{1}$ is given by [9]:

$$
K_{1}=\frac{\tan \theta(t)}{2}-\frac{\sin (\theta(t))}{6}\left[\frac{2[2-\cos (\theta(t))]+[1+\cos (\theta(t))]}{[2-\cos (\theta(t))][1+\cos (\theta(t))]}\right]
$$

and $K_{2}$ are given by [7]:

$$
K_{2}=\frac{2^{-5 / 3} \cos (\theta(t)) \sin (\theta(t))}{[1+\cos (\theta(t))]^{4 / 3}[2-\cos (\theta(t))]^{5 / 3}}
$$

Bellehumeur et al. [7] used the assumption $R_{p}(t)=R_{p}(0)$ for initial stage sintering to write an explicit ODE based on Eq. (1). The quadratic form is solved here directly which provides two symmetric solution for $d \theta / d t$. The positive solution $\left(-B+\sqrt{B^{2}+4 A}\right) /(2 A)$ is chosen here where,

$$
\begin{aligned}
& A=8\left(\lambda K_{1}\right)^{2} \\
& B=-2 \lambda K_{1}+\frac{\eta R_{p}(0)}{\sigma} \frac{K_{1}^{2}}{K_{2}} .
\end{aligned}
$$

Various relaxation times between 0.154 to $154 \mathrm{~s}$ which are equivalent to Deborah $\left(\mathrm{De} \equiv \lambda \sigma / R_{e q v}(0) \eta\right)$ numbers 1.0 to 1000 are considered. However, note that the relaxation time of the particle is not available experimentally, and only a qualitative discussion is intended here. Here to compare with the experimental results the characteristic coalescence time is defined as $R_{\text {eqv }}(0) \eta / \sigma=0.1536$ assuming $R_{\text {eqv }}(0)=33.95 \mu \mathrm{m}$ (see Section 2.2.2) and $\sigma=0.042$. Furthermore, for the Newtonian case $(\lambda=0)$ Pokluda et al. [6] model is directly solved with an improved $K_{1}$ term as suggested by Scribben 
et al. [9]. The results of these calculations is presented in Figure 10, It should firstly be emphasised that a quantitative comparison with the experimental results is not intended in this figure. For all cases, a slower coalescence rate is observed as De is increased. More interestingly, Figure 10 shows that the De number (and hence the viscoelasticity) has no impact on overall behaviour of the curves: for all cases, the neck size is strictly increasing in time. The theoretical results, clearly show that the initial delayed growth/contraction could not occur as a result of viscoelasticity. However, the viscoelasticity of the polymeric melt does change the rate of coalescence which can explain the overall slow rates for PEK HP3. Nonetheless, the neck growth remains strictly increasing.

In the next section the elongation effects will be investigated in detail to determine whether it could explain the contraction/delayed growth of coalescing PEK grains.

\section{Coalescence of elongated particles}

In this section, the simulations details are provided. Since we are interested in elongation effects, the contact orientation of the two coalescing particles becomes relevant. However, it is outside the scope of the current paper to parametrise and systematically consider different contact orientations. It has been shown that elongated grains will align themselves to the powder's flow direction during the spreading stage of the process (see Haeri et al. [1], Haeri [2]). Therefore, in this section the neck growth dynamics is investigated only for grains in head-to-head contacts. 


\subsection{Methodology and validation}

To model the coalescence, the fluid phase continuity equation and NavierStokes momentum equations are solved to predict the motion of the poly-

meric melt (assuming Newtonian behaviour) and the surrounding fluid (here assumed to be air). The continuity and Navier-Stokes equations are respectively given by

$$
\begin{gathered}
\nabla \cdot \mathbf{u}=0 \\
\rho \frac{\partial \mathbf{u}}{\partial t}+\rho \nabla(\mathbf{u u})=-\nabla P+\eta \nabla^{2} \mathbf{u}+\mathbf{F},
\end{gathered}
$$

where $\mathbf{u}$ is the velocity (vector) of the fluid and $P$ is the pressure. Here, $\mathbf{F}$ is the surface tension force which acts only on the interface between the two phases (i.e. the coalescing particles and the surrounding fluid which here is assumed to be air). To track the evolution of the air-particle interface (i.e. the neck growth rate) the volume-of-fluid ( $\mathrm{VoF})$ approach 28] is used. In this method a smooth volume fraction function $\phi$ which varies from one in the liquid region (inside the particles) to zero in the surrounding fluid is defined which evolves according to

$$
\frac{\partial \phi}{\partial t}+\mathbf{u} \cdot \nabla \phi=0
$$

Note that the viscosity and density defined in Eqs. (8) and (9) are mixture properties. For example the density is defined by $\rho=\phi \rho_{p}+(1-\phi) \rho_{a}$, where $\rho_{p}$ is the particle density and $\rho_{a}$ is the air density. Consequently, the surface tension force can be calculated as [28]

$$
\mathbf{F}=-\sigma\left[\nabla \cdot\left(\frac{\nabla \phi}{|\nabla \phi|}\right)\right] \nabla \phi
$$


However, here the surface tension force calculation is improved by using a level-set function to reduce the spurious currents on the interface, see [29] for more details. To validate the simulations the coalescence of two spherical grains are compared against the theoretical results of the modified Pokluda model (see [9] and Eq. (4) for details).

The computational domain is a cube with unit edge length and is discretised using two different grid levels. The fine grid consists of $352 \times 249 \times 249$ and the coarse grid has $220 \times 170 \times 170$ points which are clustered near the centre to provide 133 and 80 grid points along the diameter of each particle with a uniform spacing of $h=6.02 \times 10^{-4}$ and $h=0.001$ respectively. This resolves the coalescence with a high accuracy while the boundaries of the computational domain are far enough to ensure the simulations are independent of the boundary conditions. The maximum CFL number during the simulation remains below one which also ensures a time step size smaller than the capillary step size requirements which is calculated to be $\Delta t_{\sigma}=7.3 \times 10^{-4}$. Figure 11 shows the simulation set-up, grid resolution around the coalescing particles and the various aspect ratios used for the computer experiments in Section 4.2 .

Figure 12 shows the simulation results. During the discussion the parameter $R_{n} / R_{p}(0)$ will be referred to as the neck size. However, after the coalescence is complete, $R_{n} / R_{p}(0)$ represents the non-dimensional diameter of the particles along the line that the neck was originally growing. The simulations are grid independent and the difference in the neck growth rate up to around $t^{*}=21$ is negligible and the overall characteristics of the curves remain similar.

Compared to the simulations, the theory predicts a faster initial dynamics particularly between $t^{*}=0$ and $t^{*}=5$. This seems to be the result 
of the analytical description of the neck region used for derivation of the theory. The assumed geometrical evolution is presented in Figure 9a. The neck region is highlighted with a red box in this figure, and evidently in the theory a sharp curvature throughout the evolution is retained. The initial condition for the numerical simulation, similar to the theory, is that of a cusp. However, the numerical scheme predicts a smoother curvature at the neck region during the evolution, see Figure $9 \mathrm{~b}$,

Jagota et al. [30] discussed two different modes of sintering: an initial elastic adhesion or the "zipping mode" growth and viscous sintering or "stretching mode" growth. The available theories assume an analytical function for the evolution of $\theta$ (see Figure 9 which mimics the zipping mode throughout the entire solution despite the fact that they actually attempt to model the viscose sintering (i.e. the stretching mode). However, the simulations consistently simulate the stretching mode. Furthermore, Eggers et al. [12] showed that the external fluid has a great impact on the evolution of the neck as well. The presence of an external fluid significantly increases the length-scale of the local curvature even for very large relative viscosities (i.e. $\lambda \gg 1$ ), whereas all the available theoretical results assume $\lambda=\infty$ (i.e. no external fluid). Nonetheless, the growth rates are qualitatively in agreement until the coalescence is complete at $t^{*} \approx 21$.

\subsection{Grain shape effects}

The simulations are run on a scaled computational domain to ensure numerical stability but the physical properties are also scaled such that the non-dimensional coalescence time $t^{*}=(t \sigma) /\left(R_{p}(0) \eta\right)$ has the same order as that of the PEK HP3. However, in this section the characteristic time is increased to $\left.R_{e q v}(0) \eta / \sigma=0.33\right)$ where $R_{e q v}(0)$ is the radius of a sphere with 
the same surface area of the initial ellipsoidal particle which is approximated (by equating the area of a sphere $S_{\mathrm{sph}}=4 \pi R^{2}$ to the approximated area of an ellipsoid) as

$$
R_{e q v}(0)=\sqrt{\left(\frac{(a b)^{p}+(a c)^{p}+(b c)^{p}}{3}\right)^{1 / p}} .
$$

In Eq. 12, $a=b \mathrm{~A}_{r}=c \mathrm{~A}_{r}$ is the major semi-axis of the ellipsoid and $p=$ 1.6. It is also worth mentioning that choosing to increase the characteristic time slows the dynamics, however, it also allows increasing the time step of the simulation and overall lowers the computational cost. It is clear that this only changes the scaling and not the nature of the neck growth curves and hence is adequate for the present parametric investigation of the grain shape.

Figure 13 shows the results of these simulations for various aspect ratios. Firstly, note that the neck growth rate curves resemble the trends observed in experiments. Although the results are not quantitatively comparable to the experiments, a similar delayed growth rate is observed in the simulations for aspect ratio $\mathrm{A}_{r}=1.75 \cdots 2.25$. Furthermore, the rate of growth becomes slower compared to a sphere with the similar surface area even for aspect ratios as small as $\mathrm{A}_{r}=1.25$.

The surface tension forces act to reduce the surface area as explained before. The results here do not contradict this principle. Figure 14 shows the evolution of the total surface area of the two particles during the binary coalescence. All the curves show a monotonic decrease in the total surface areas. However, a minimum is observed at the tail of the curves which is related to the deformation of the melted particles in the transverse direction (the y-direction) after the coalescence is complete (i.e. when a single large particle is achieved). Such oscillation is expected and will damp out 
by viscous dissipation if the simulation is continued [31]. The monotonic decrease of the total surface area confirms the surface area minimisation principle and is observed even for the case with $\mathrm{A}_{r}=2.5$. At $\mathrm{A}_{r}=2.5$ the particles separate and this state (two separate spheres) appears to be a local minimum. In the next section, we will show that this state is reached as the result of the local curvatures on the surface which is a direct consequence of the topology of the particles.

\subsection{Local curvature analysis}

The surface tension forces are directly proportional to the curvature on the surface of the particles $(F=\sigma \kappa \mathbf{n}$, were $\mathbf{n}$ is the unit normal to the interface). Compared to the coalescence of two spherical particles, the topology and consequently the local variations in curvature are expected to have a great impact on the coalescence of non-spherical particles, which will be investigated next.

\subsection{1. post-processing procedure}

To calculate the curvature first an iso-surface of a constant volume fraction of the liquid is required. This can be extracted from the simulations at a specific volume fraction. The inset in Figure 11 shows such surfaces. In this study, all the iso-surfaces are extracted at $\phi=0.5$. However, this surface is very rough since it is extracted from a 3D discrete dataset (i.e. the computational grid). To calculate the curvature second derivatives of the parametrised iso-surface are required. However, significant fluctuations due to the roughness of the extracted iso-surfaces make the comparison impossible.

However, if the boundaries of $\phi=0.5$ iso-surfaces are projected onto the xy-plane (see Figure 11) a 2D geometrical curve will be obtained (inset of 
Figure 15). The lower half of the perimeter of these projections for all $\mathrm{A}_{r}$ and at a specific time $t=1.6$ are presented in Figure 15 . The time $t=1.6$ is just before the separation of two particles with $\mathrm{A}_{r}=2.5$. It is worth mentioning that $t=1.6$ is not non-dimensionalised since it is a particular event relevant to the coalescence of two particles with $\mathrm{A}_{r}=2.5$. Also in this figure, the $\mathrm{x}$ - and $\mathrm{y}$-coordinates are normalised by the semi-major and minor axes (i.e. $a$ and $b$ in Figure 11). The analysis is limited to these 2D curves which is justified since an axis of symmetry exists around " $\mathrm{x}$ ". The curvature can be calculated using

$$
\kappa=\frac{x^{\prime} y^{\prime \prime}-y^{\prime} x^{\prime \prime}}{\left(x^{\prime 2}+y^{\prime 2}\right)^{1.5}}
$$

assuming $x$ and $y$ are parametrisation of the curve with respect to a variable $t$ with equal spacing. To smooth the curvature values to enable comparison, $N_{p}$ points are equally chosen from the range of values in $x$ which are referred to as $x_{I}$ here. The $y_{I}$ values are determined by linear interpolations. The derivatives are calculated by using $x_{I}$ and $y_{I}$ and a Gaussian filter is applied to the calculated values, finally $\kappa$ is calculated using Eq. 13 with ${\tilde{x_{I}}}^{\prime}, \tilde{y}_{I}^{\prime}$, ${\tilde{x_{I}}}^{\prime \prime}$ and ${\tilde{y_{I}}}^{\prime \prime}$, where $\tilde{*}$ is a filtered value.

\subsubsection{Analysis}

Figure 16 shows the results of these calculations at different time steps. The results are presented for $\mathrm{A}_{r}=1.0,1.5,2.0$ and 2.5 only, however, similar trends are observed for other aspect ratios. In addition, The evolution of curvature for the spherical particles is labelled with $\mathrm{A}_{r}=1.0$ in Figure 16 . For the case of coalescence of two spheres, a very large initial curvature at the contact point is observed which derives the coalescence. During the coalescence, the contact point flattens and the curvature of the neck region 
approaches zero. However, the width of the neck has grown by this time substantially, and a wide region with a curvature near zero can be observed. After this stage, the coalescence is driven primarily by the small curvature on the two ends of a monolithic dumbbell-shaped particle.

A similar trend is observed for $\mathrm{A}_{r}=1.5$ and 2.0, however firstly, the curvature on the contact point is smaller, and secondly, the width of the flattened neck region remains much smaller compared to the coalescence of two spheres. However, still similar trends are observed and although the dynamic is slower due to the smaller initial curvature at the contact point, eventually the coalescence completes.

At the critical aspect ratio of $\mathrm{A}_{r}=2.5$, however, an initial growth is observed but eventually, the growth stops and two small regions at the contact point of the neck with the main bodies form. The local curvature assumes a minimum at these two points (identified by two arrows in Figure 16). These two large negative curvatures (local minima) then force the shrinkage of the neck region and eventually cause the separation of the two particles.

\section{Conclusion}

In this paper, the coalescence of PEK HP3 powder grains which are extensively used in the LS process are considered. Particle characterisation was performed and the rate of neck growth was determined for these particles using image analysis techniques. An initial delay and in some cases, a contraction was observed in the neck growth. To investigate this phenomenon further, the dynamics of a single particle was investigated experimentally. The single particle experiments show that the particles progressively achieve a spherical shape supported by the aspect ratio values, which changes the 
coalescence dynamics.

However, viscoelasticity can also alter the sintering behaviour. Therefore, these factors were investigated in isolation. The effects of viscoelasticity was studied by considering the sintering of spherical pairs of particles. It is shown that the viscoelasticity can decrease the rate of coalescence however, the growth remains strictly increasing. To isolate the shape effects VoF simulations were performed on ellipsoidal particles with aspect ratios ranging from 1.0 (sphere) to 2.5. The rate of neck growth was assessed and it is found that the particles can separate at large aspect ratios and a critical aspect ration of $\mathrm{A}_{r}=2.5$ is determined.

It is shown that the separation is related to the topology of the coalescing units and the local curvature values. At the critical aspect ratio, it is found that two local minima at the contact points of the neck with the main body of the particles form which force a local shrinkage in the neck and eventually the two particles separate.

\section{Acknowledgement}

Supercomputing time on ARCHER is provided by the "UK Consortium on Mesoscale Engineering Sciences (UKCOMES)" under the UK Engineering and Physical Sciences Research Council (EPSRC) Grant No. EP/R029598/1 and Resource Allocation Panel (RAP) Grant E504. The authors also gratefully acknowledge the use of Cirrus HPC Service (http://www.cirrus.ac.uk) funded by the University of Edinburgh and EPSRC (EP/P020267/1) which allowed us to complete this work. 


\section{Data availability}

The raw data are retained on the UK Research Data Facility (http://rdf.ac.uk) and can be shared upon request. The processed data are available to download from http://dx.doi.org/10.15129/7bf114fc-a3ce-4036-ae84-54b6c0ae7131.

\section{References}

[1] S. Haeri, Y. Wang, O. Ghita, J. Sun, Discrete element simulation and experimental study of powder spreading process in additive manufacturing, Powder Technology 306 (2017) 45-54.

[2] S. Haeri, Optimisation of blade type spreaders for powder bed preparation in additive manufacturing using dem simulation, Powder Technology 321 (2017) 94-104.

[3] W. Nan, M. Pasha, T. Bonakdar, A. Lopez, U. Zafar, S. Nadimi, M. Ghadiri, Jamming during particle spreading in additive manufacturing, Powder Technology 338 (2018) $253-262$.

[4] J. Frenkel, Viscous flow of crystalline bodies under the action of surface tension, Journal of Physics 9 (1945) 385.

[5] J. Eshelby, Discussion in paper by shaler, seminar on the kinetics of sintering, Metals Trans 185 (1949) 806-807.

[6] O. Pokluda, T. Bellehumeur, J. Machopoulos, Modification of frenkel's model for sintering, AIChE Journal 43 (1997) 3253-3256.

[7] C. T. Bellehumeur, M. Kontopoulou, J. Vlachopoulos, The role of viscoelasticity in polymer sintering, Rheol Acta (1998). 
[8] D. D. Joseph, Fluid dynamics of viscoelastic liquid, Springer, 1990.

[9] E. Scribben, D. Baird, P. Wapperom, The role of transient rheology in polymeric sintering, Rheol. Acta 45 (2006) 825-839.

[10] H. Xie, A geometrical model for coalescing aerosol particles, Journal of Aerosol Science 39 (2008) 277-285.

[11] M. L. Eggersdorfer, D. Kadau, H. J. Herrmann, S. E. Pratsinis, Multiparticle sintering dynamics: From fractal-like aggregates to compact structures, Langmuir 27 (2013) 6358-6367.

[12] J. Eggers, J. R. Lister, S. H. A., Coalescence of liquid drops, Journal of Fluid Mechanics 401 (1999) 293-310.

[13] R. W. Hopper, Coalescence of two equal cylinders: exact results for creeping viscous plane flow driven by capillarity, J. Am. Ceram. Soc. 67 (1984) C262-C264.

[14] A. Jagota, P. R. Dawson, Simulation of the viscous sintering of two particles, Journal of the American Ceramic Society (1990).

[15] J. I. Martinez-Herrera, J. J. Derby, Viscous sintering of spherical particles via finite element analysis, Journal of the American Ceramic Society 78 (1995) 645-649.

[16] V. Yadha, J. Helble, Modeling the coalescence of heterogenous amorphous particles, Journal of Aerosol Science 35 (2004) 665-681.

[17] H. H. Kart, G. Wang, I. Karaman, T. Cagin, Molecular dynamics study of the coalescence of equal and unequal sized cu nanoparticles, International Journal of Modern Physics C 20 (2009) 179-196. 
[18] F. Hussain, S. Hayat, M. Imran, S. Ahmad, F. Bouafia, Sintering and deposition of nanoparticles on surface of metals: A molecular dynamics approach, Computational Materials Science 65 (2012) 264-268.

[19] M. J. Kirchhof, H.-J. Schmid, W. Peukert, Three-dimensional simulation of viscous-flow agglomerate sintering, Physical Review E 80 (2009) 026319 .

[20] C. Balemans, N. O. Jaensson, M. Hulsen, P. Anderson, Temperaturedependent sintering of two viscous particles, Additive Manufacturing (2018).

[21] M. Kamyabi, R. Sotudeh-Gharebagha, R. Zarghamia, K. Saleh, Principles of viscous sintering in amorphous powders: A critical review, Chemical Engineering Research and Design 125 (2017) 328-347.

[22] E. E.-M. Solutions, EOS PEEK HP3, the high-grade performer, Technical Report, EOS, 2008.

[23] S. Berretta, K. E. Evans, O. R. Ghita, Predicting processing parameters in high temperature laser sintering (ht-ls) from powder properties, Materials \& Design 105 (2016) 301-314.

[24] G. M. Vasquez, C. E. Majewski, B. Haworth, N. Hopkinson, A targeted material selection process for polymers in laser sintering, Additive Manufacturing 1-4 (2014) 127-138.

[25] M. Vasquez, B. Haworth, N. Hopkinson, Methods for quantifying the stable sintering region in laser sintered polyamide-12, Polymer Engineering and Science 53 (2013) 1230-1240. 
[26] L. Benedetti, B. Brulé, N. Decraemer, K. E. Evans, O. Ghita, Evaluation of particle coalescence and its implications in laser sintering, Powder Technology (2019).

[27] O. R. Ghita, E. James, R. Trimble, K. E. Evans, Physico-chemical behaviour of poly (ether ketone) (pek) in high temperature laser sintering (ht-ls), Journal of Materials Processing Technology 214 (2014) 969-978.

[28] S. Haeri, S. H. Hashemabadi, Three dimensional cfd simulation and experimental study of power law fluid spreading on inclined plates, International Communications in Heat and Mass Transfer (2008).

[29] A. Albadawi, D. Donoghue, A. Robinson, D. Murray, Y. Delaure, Influence of surface tension implementation in volume of fluid and coupled volume of fluid with level set methods for bubble growth and detachment, International Journal of Multiphase Flow 53 (2013) 11-28.

[30] A. Jagota, C. Argento, S. Mazur, Growth of adhesive contacts for maxwell viscoelastic spheres, J. Appl. Phys. 83 (1998) 250-259.

[31] A. M. Soto, T. Maddalena, A. Fraters, D. va der Meer, D. Lohse, Coalescence of diffusively growing gas bubbles, Journal of Fluid Mechanics 846 (2018) 143-165.

[32] Victrex, High Performance Polymers Victrex HT G22, Technical Report, Victrex, 2014.

[33] Y. Wang, D. Rouholamin, R. Davies, O. Ghita, Powder characteristics, microstructure and properties of graphite platelet reinforced poly ether 
ether ketone composites in high temperature laser sintering (ht-ls), Materials \& Design 88 (2015) 1310-1320. 


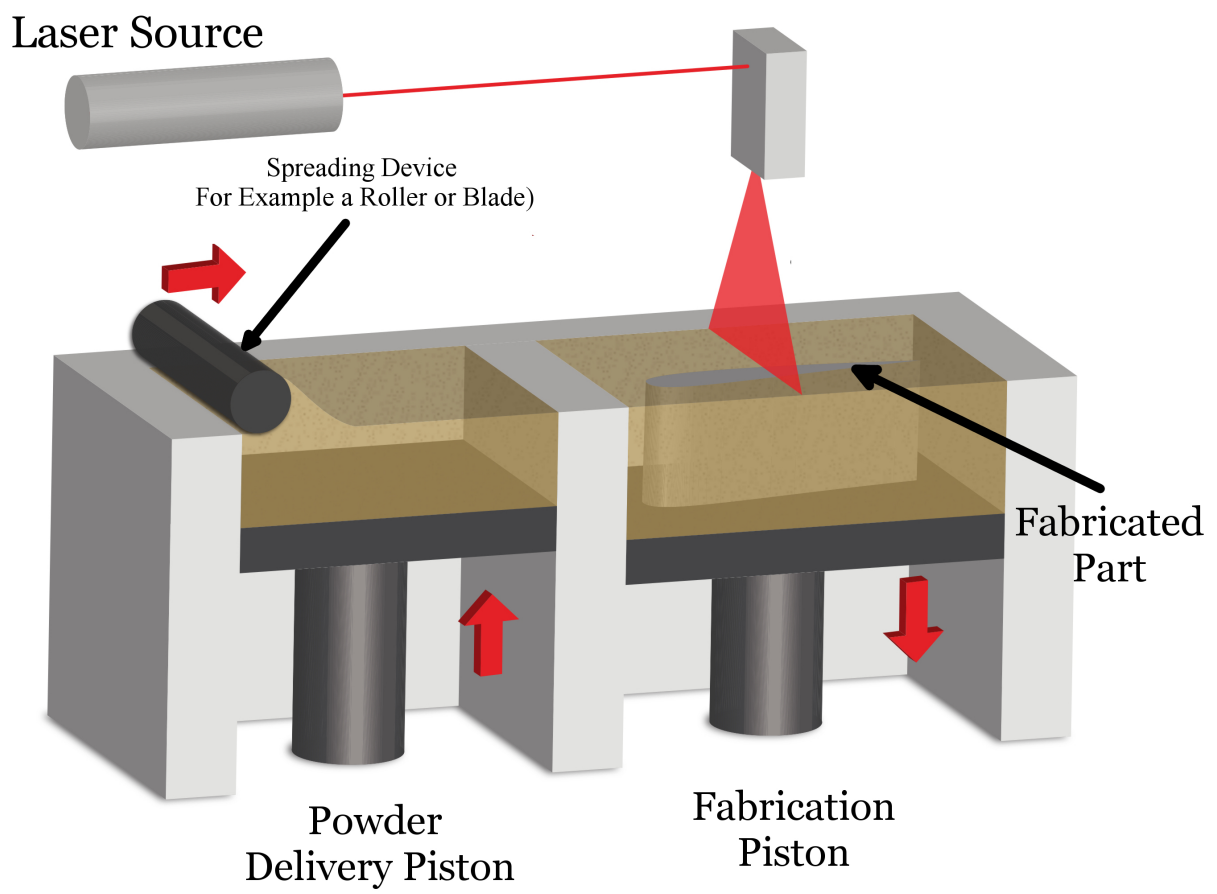

Figure 1: Schematic Presentation of the Laser Sintering Process 


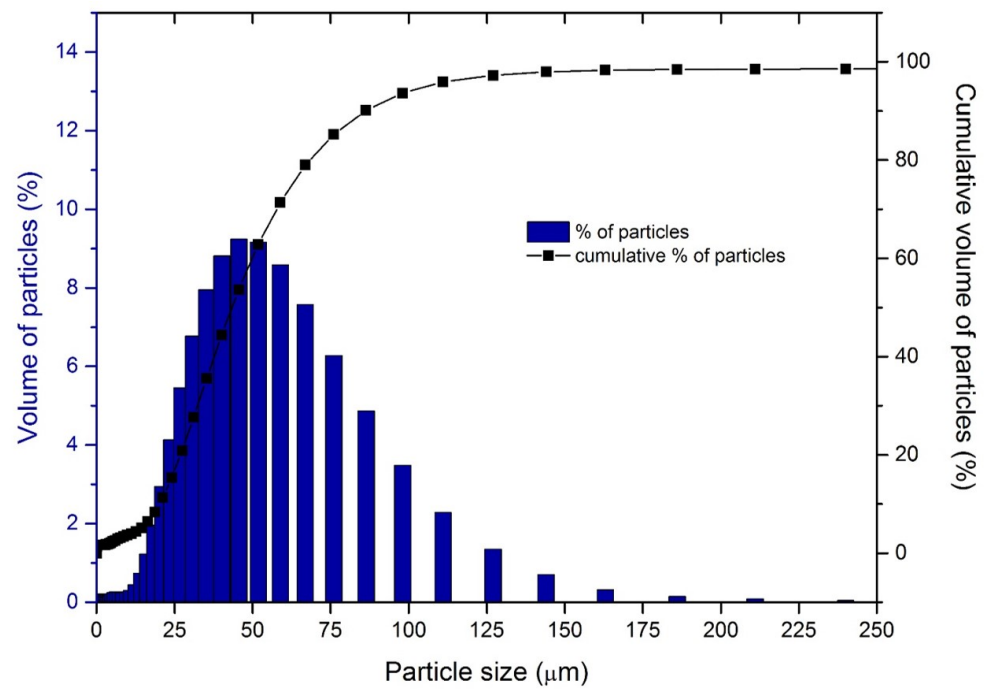

Figure 2: Particle size distribution for PEK HP3. 

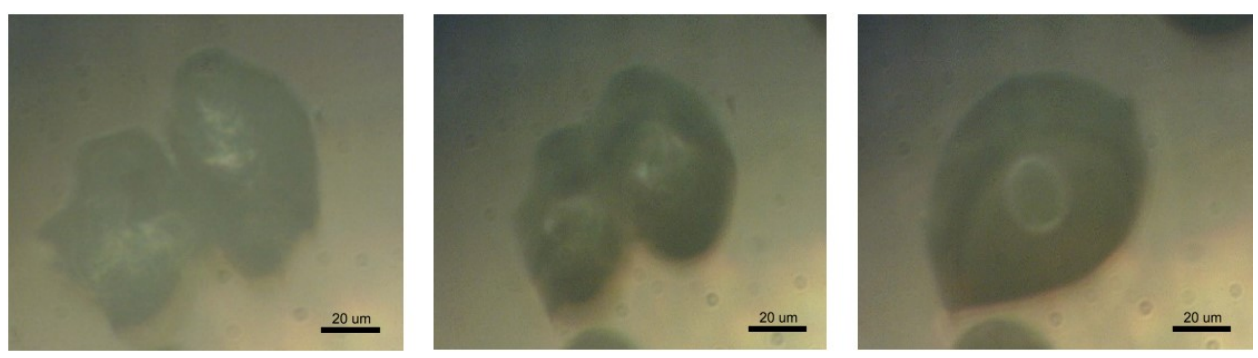

Figure 3: Coalescence of PEK HP3 particles where particles attain a final smooth spherical shape. The figures correspond to particles in room temperature, $t^{*}=0$ and $t_{\mathrm{end}}^{*}$ from left to right. 


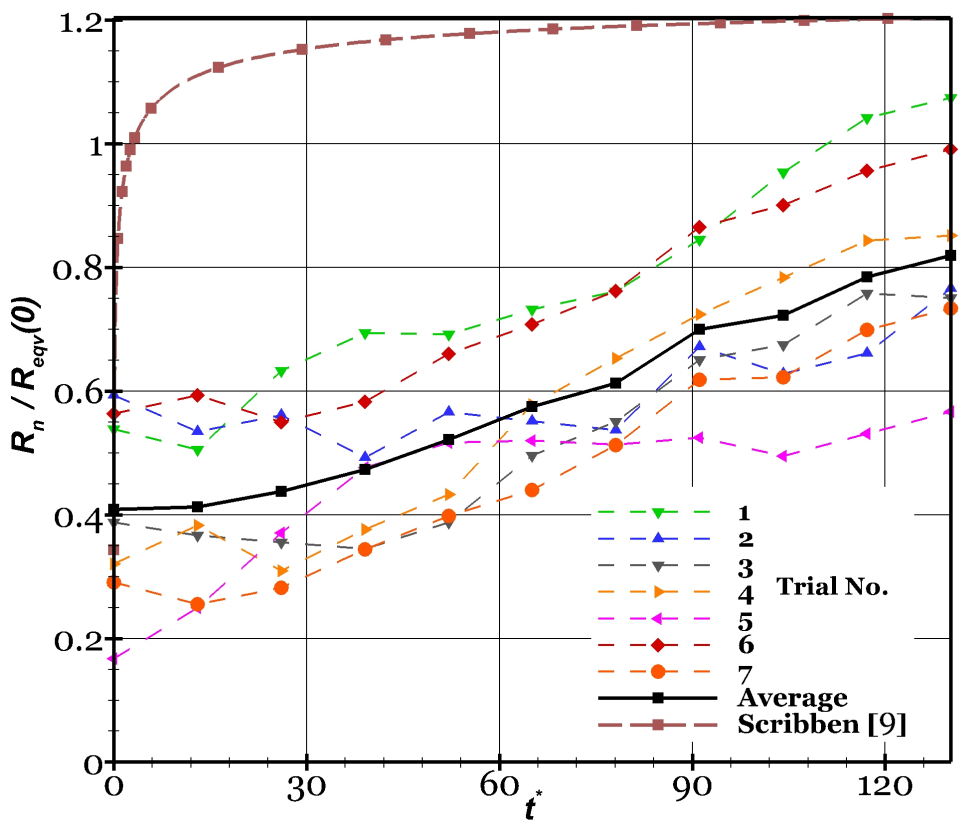

Figure 4: Progress of neck growth for PEK HP3 particles on glass substrate. Improved Pokluda model [6] is used (see [9] and Eq. (4)). 


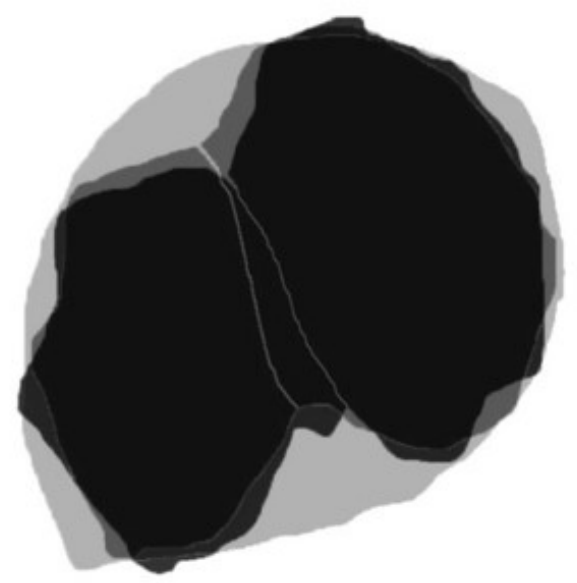

Figure 5: Progress of PEK HP3 particle coalescence. The darkest image corresponds to the particles at room temperature, followed by the particles at $t^{*}=0$ and the lightest colour corresponds to the particles at $t_{\mathrm{end}}^{*}$. 

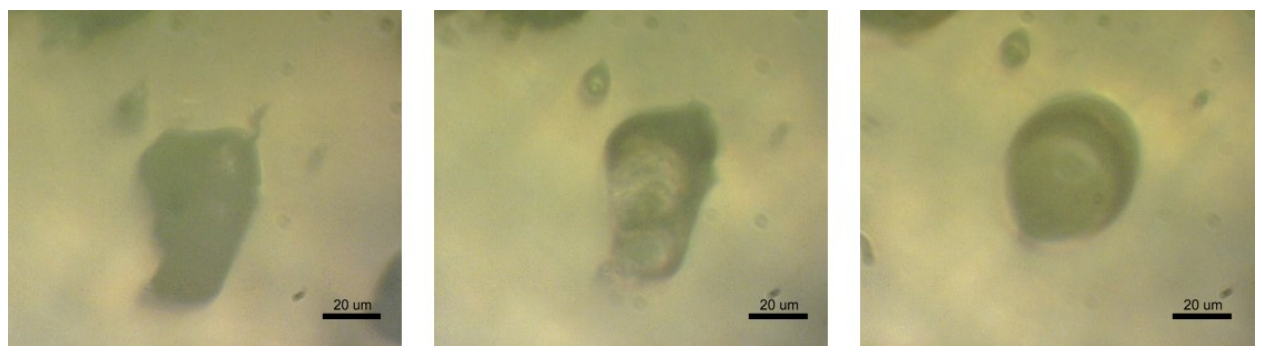

Figure 6: Hot Stage Microscospy images for individual particles of PEK HP3. An individual unit of coalescence evolves in time to minimise the surface area. The surface roughness elements flatten and particle reaches an overall spherical shape. The figures correspond to room temperature, $t^{*}=0$ and $t_{\text {end }}^{*}$ from left to right. 


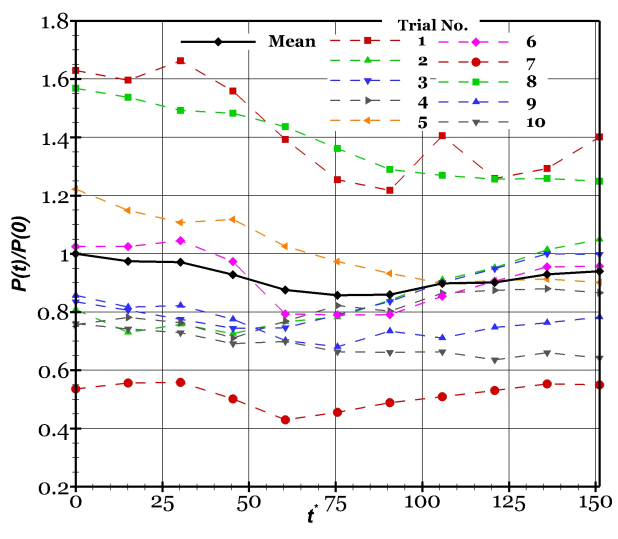

(a)

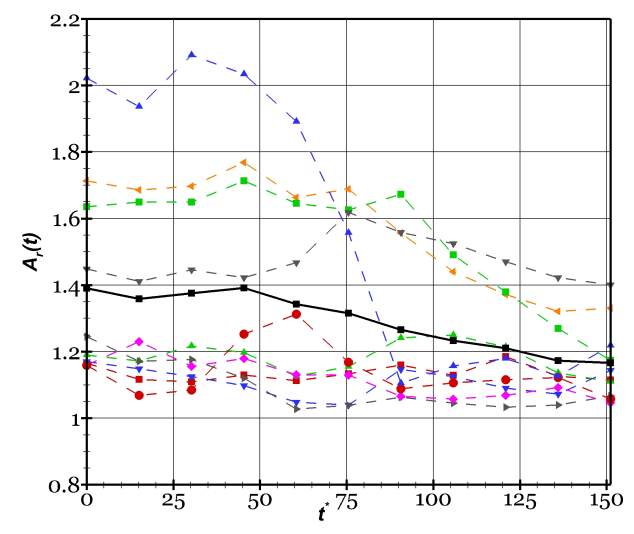

(b)

Figure 7: Evolution of individual PEK HP3 particles on glass substrate. Figure 7a shows the evolution of particle's perimeter in time; the final perimeter reduces by approximately $9 \%$ as a result of becoming more spherical. However, slight expansion is observed after $t^{*}=90$ which is believed to be caused by the loss of porosity and thermal expansion. Figure $7 \mathrm{~b}$ shows the monotonic decrease of aspect ratio towards $A_{r}=1$ (i.e. becoming more spherical). 


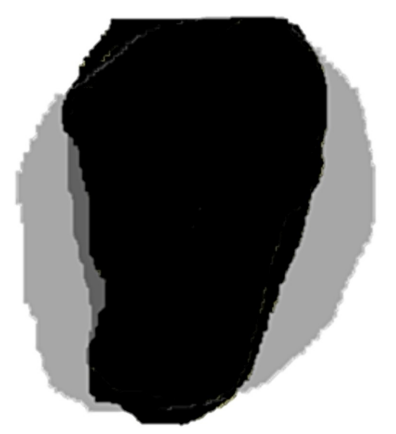

Figure 8: Changes in particle shape with temperature for PEK HP3. The darkest image corresponds to the particle at room temperature, followed by the particle at $t^{*}=0$ and the lightest colour corresponds to the particle at $t_{\mathrm{end}}^{*}$ 


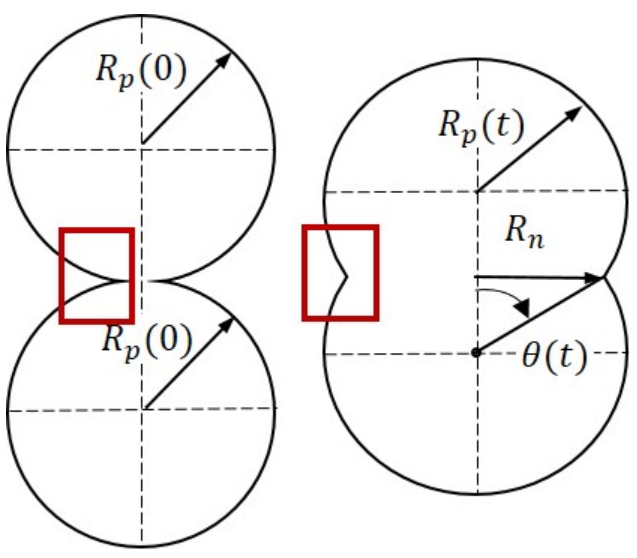

(a) Analytical
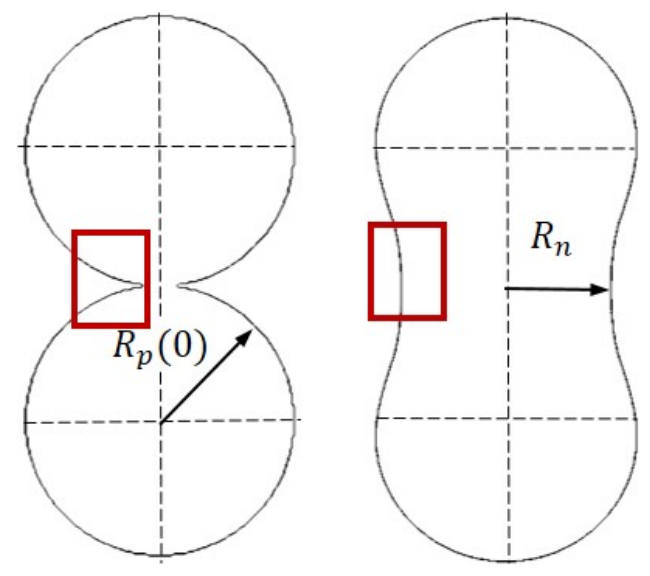

(b) Simulation

Figure 9: The evolution of the neck profile during the coalescence using the theory and numerical simulations. The analytical function that determines the neck growth in the theory, is equivalent to the zipping mode growth over the entire neck growth time (see the red boxes in Figure 9a. However, the numerical simulations consistently model the viscous sintering driving neck growth in stretching mode (the red boxes in Figure $9 \mathrm{~b}$. 


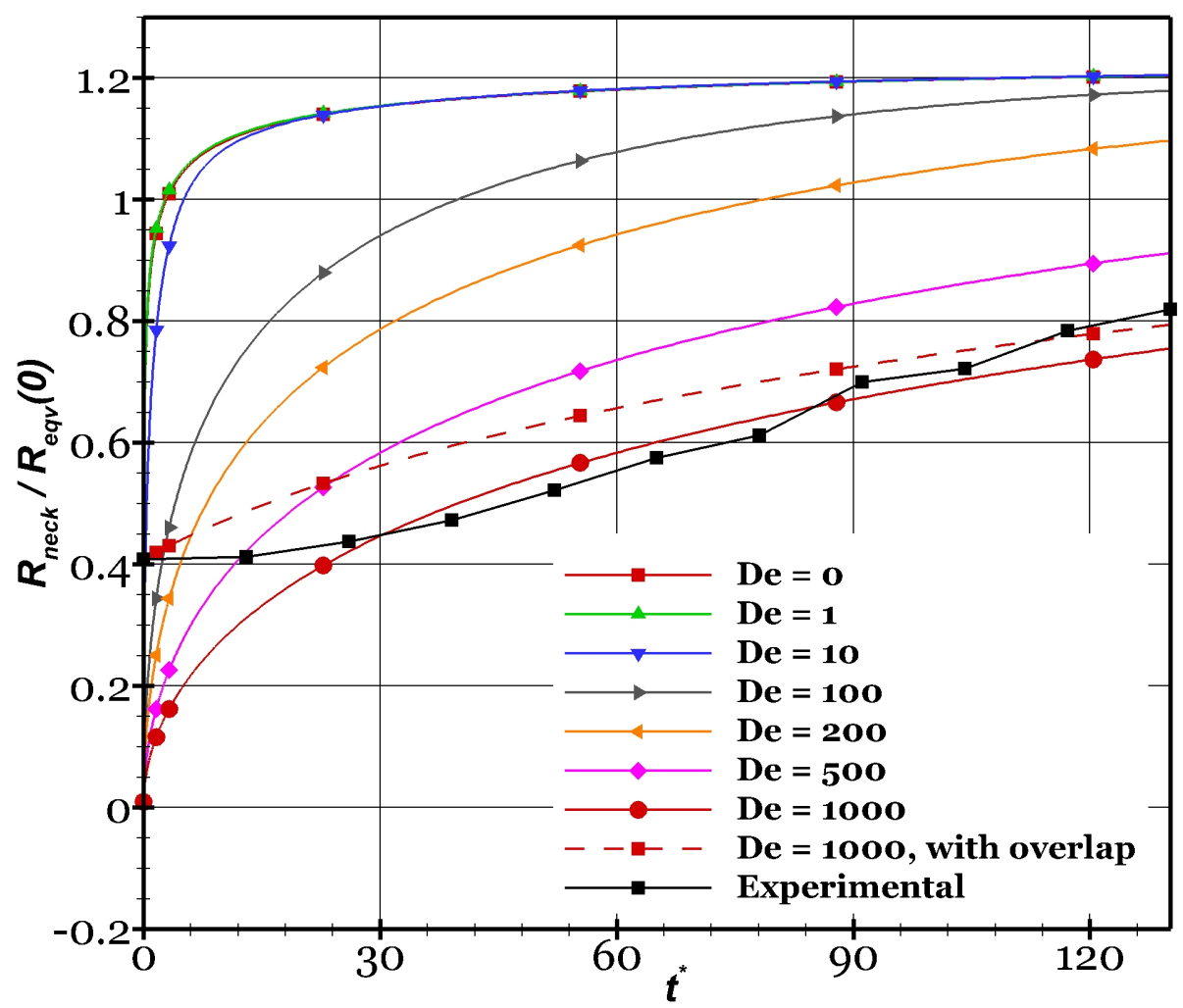

Figure 10: Effects of viscoelasticity on the neck growth rates. By increasing De, a slower rate of growth is observed. However, even for the largest De and considering the large initial overlap, the theory still predicts a monotonic increase in the neck size. Improved Pokluda et al. 6] and Bellehumeur et al. 7] models are used for $D e=0$ and $D e>0$ respectively (see Section 3 and [9] for details). 


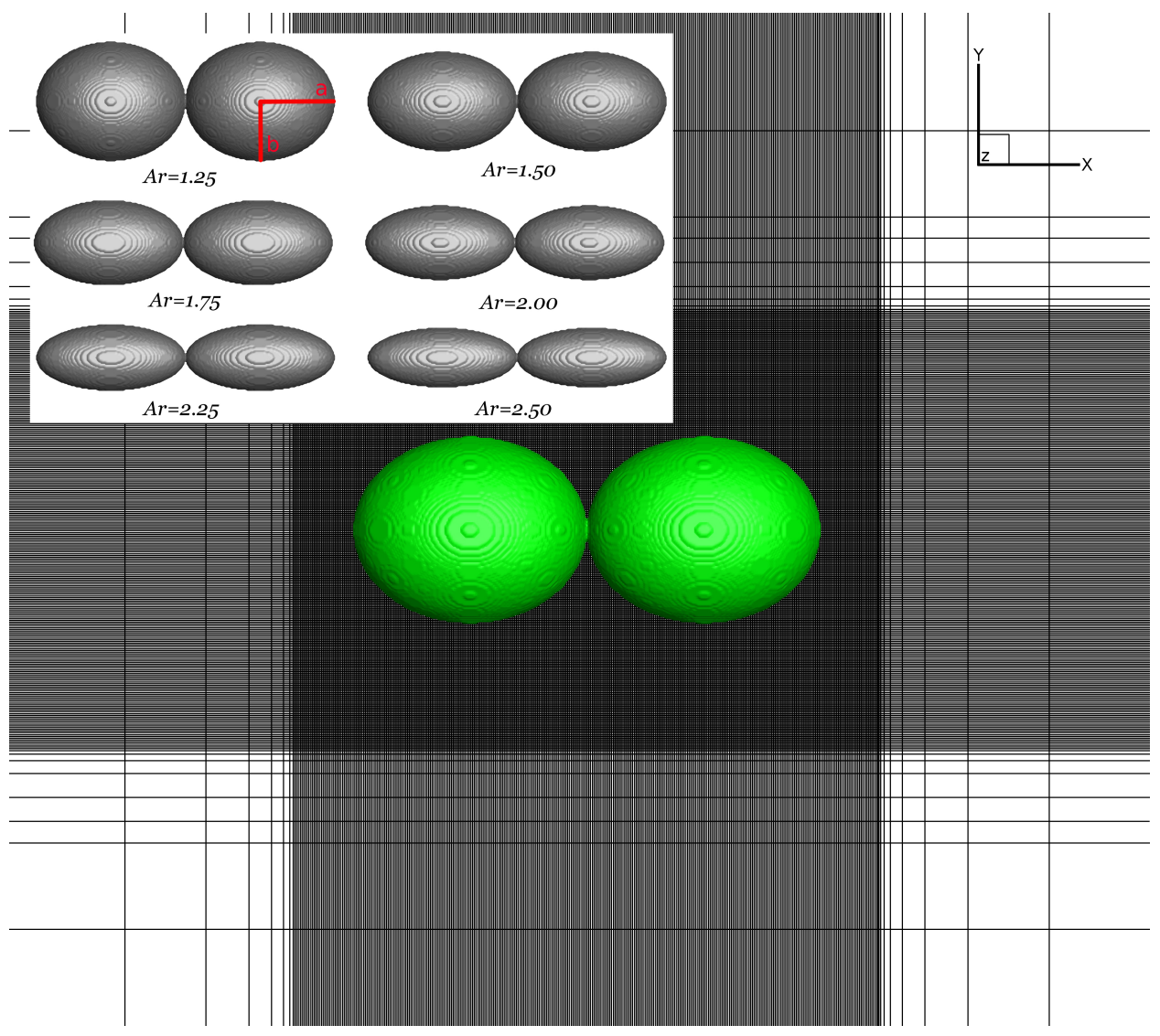

Figure 11: The simulation set-up. The grid resolution around coalescing particles is chosen to provide 80 grid point along the major axis. The different aspect ratios used for the simulations are also presented in this figure. The length of the major axis is kept the same and aspect ration is changed by changing the minor axis of the ellipsoids. 


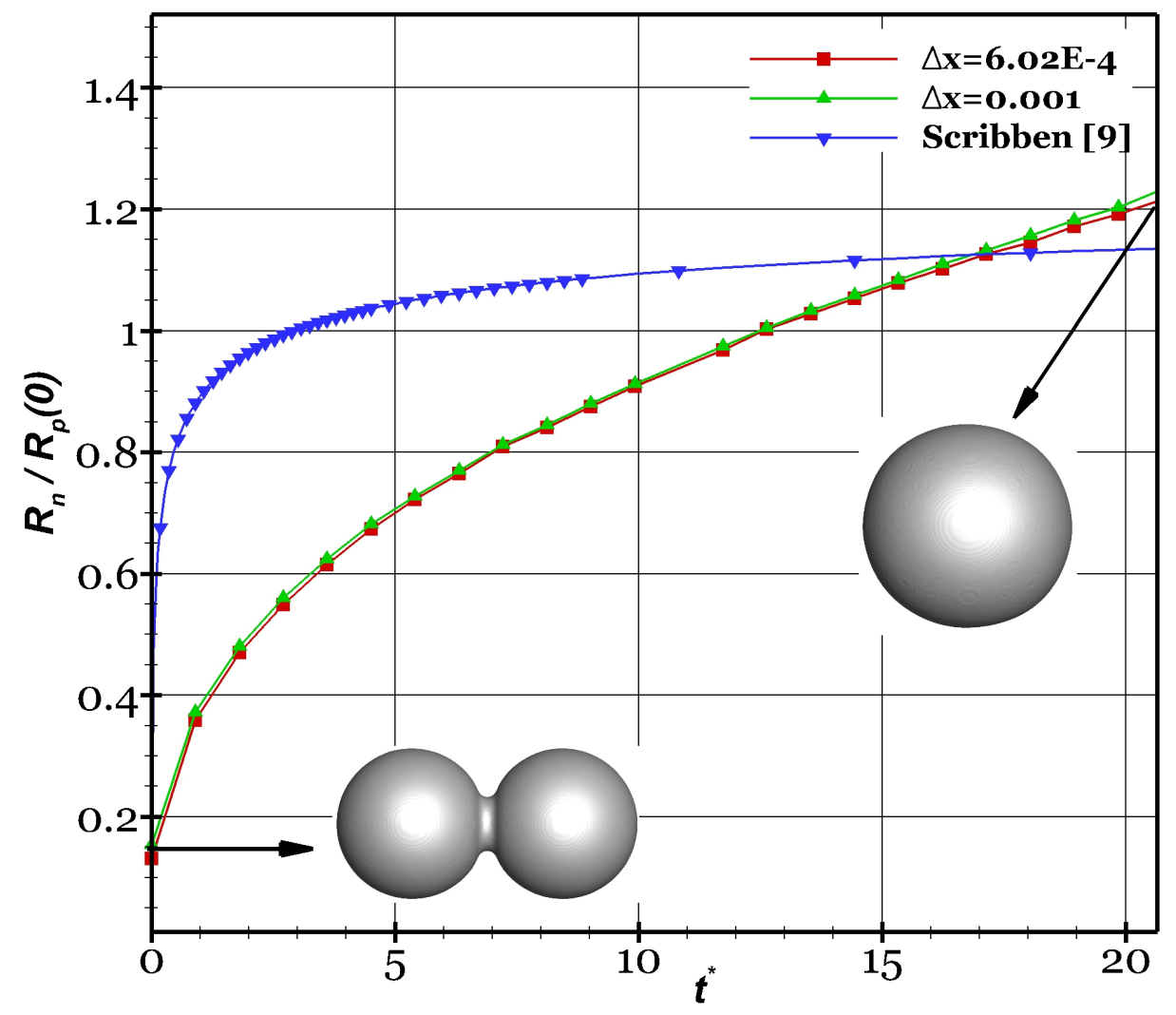

Figure 12: Validation of the VoF solver against the improved Pokluda sintering theory (see Scribben 9] and Eq. 44). The grid independence is demonstrated on two grid levels which provide 80 and 133 grid points along the diameter of each particle (sintering unit). 


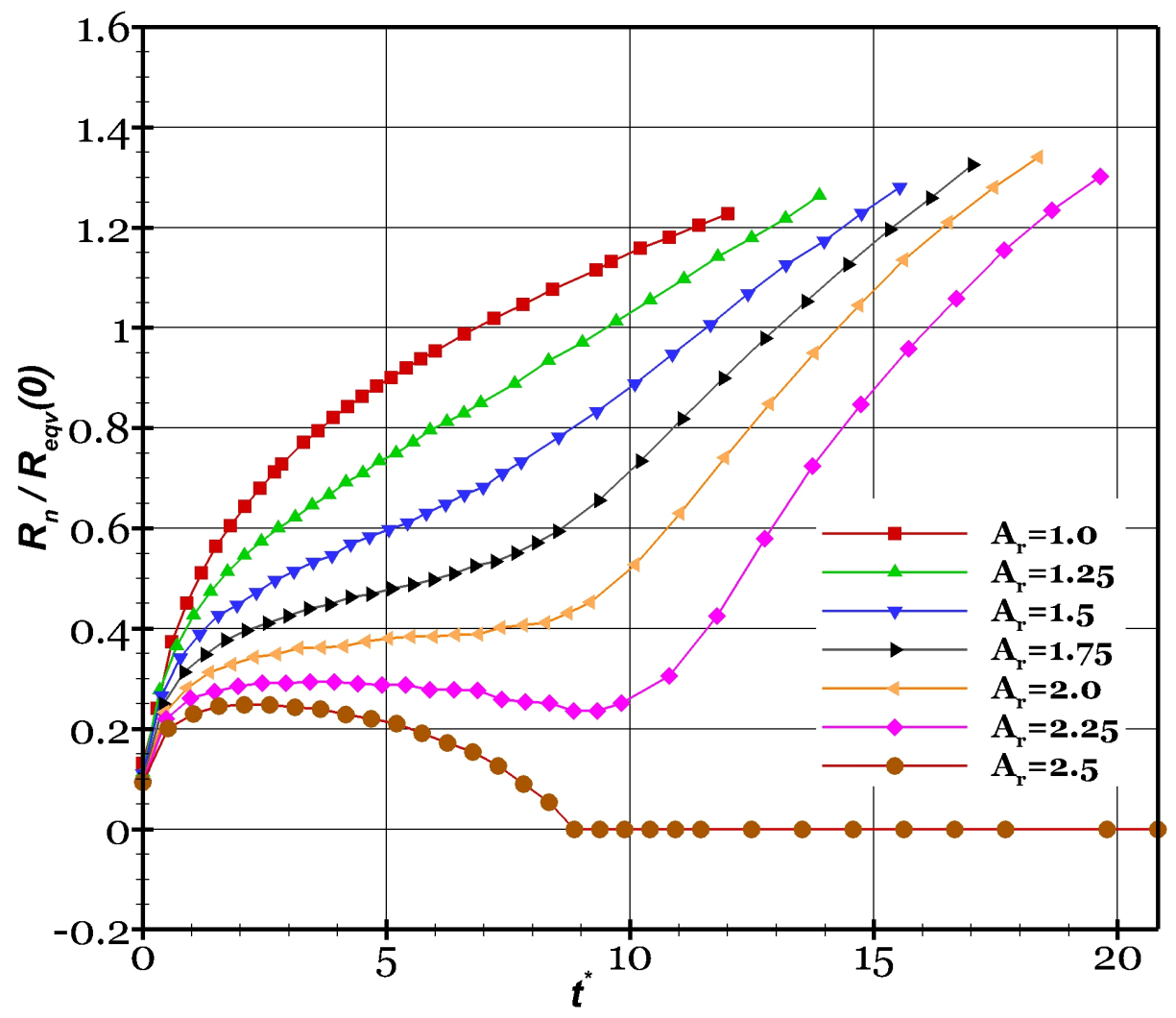

Figure 13: The neck growth rates of two ellipsoidal particles in head-to-head contact with $\mathrm{A}_{r}=1 \cdots 2.5$. For the largest aspect ratio of $\mathrm{A}_{r}=2.5$, the particles eventually separate. 


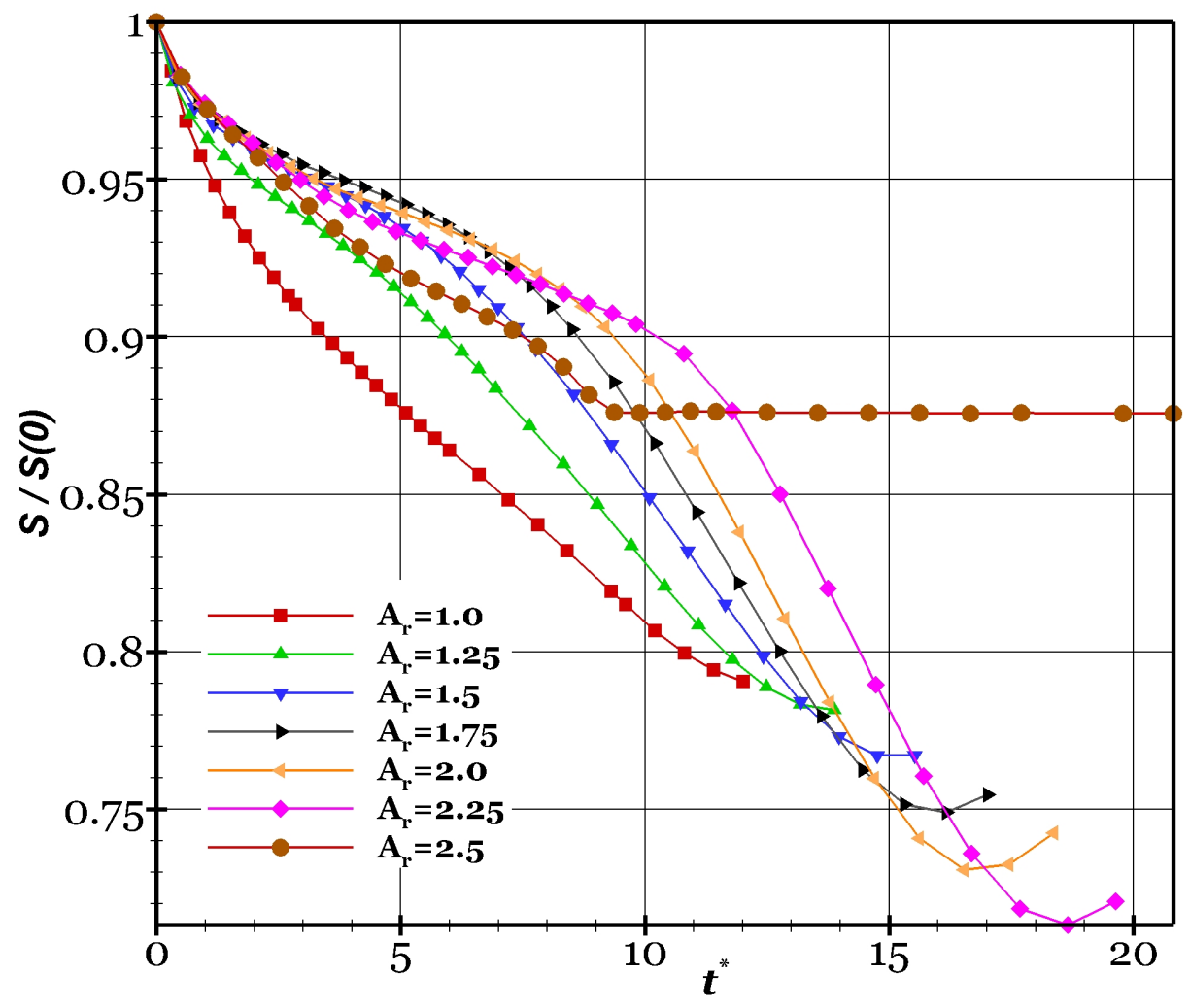

Figure 14: The evolution of the total surface areas of two ellipsoids during coalescence. 


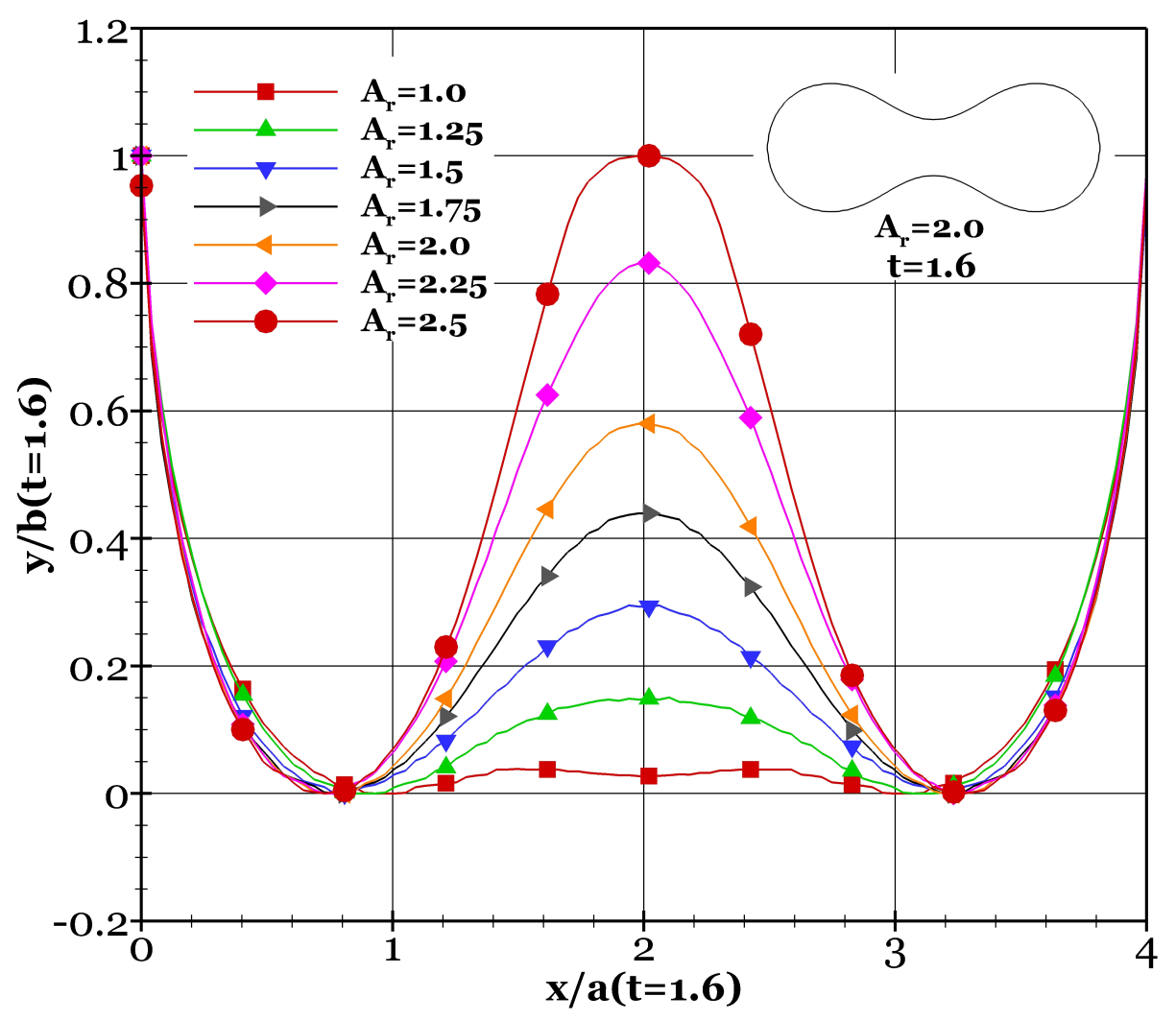

Figure 15: The curves obtained by projecting the $\phi=0.5$ iso-surfaces (see the inset of Figure 11 on the zx-plane. Only the lower half of the curve is presented with a few points along the curve. Also, all curves are plotted at $t=1.6$ just before the separation of the two particles with $\mathrm{A}_{r}=2.5$. The values $\mathrm{a}$ and $\mathrm{b}$ are the semi-major and minor axes of the ellipsoid at $t=1.6$ 

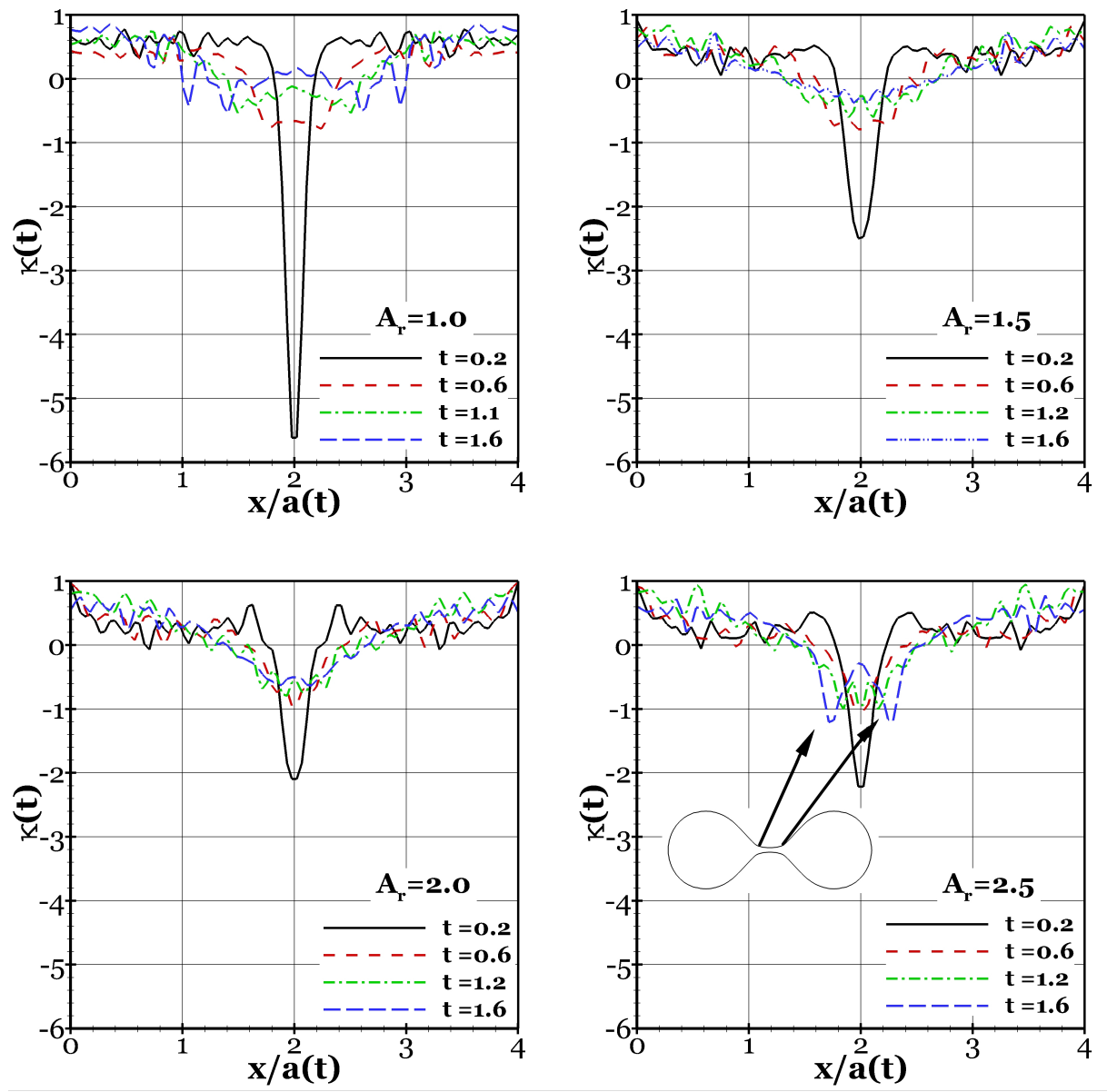

Figure 16: The evolution of the local curvature of coalescing particles for different aspect ratios. The plot for $\mathrm{A}_{r}=2.5$ just before the separation is highlighted by a pair of arrows. Two local minima at the connection point between the neck and the coalescing units is observed. 
Table 1: General information of PEK grades used in this study.

\begin{tabular}{lccc}
\hline Grade & Viscosity [Pa.s $]$ & $\mathrm{T}_{\mathrm{g}}\left[{ }^{\circ} \mathrm{C}\right]$ & $\mathrm{T}_{\mathrm{m}}\left[{ }^{\circ} \mathrm{C}\right]$ \\
PEK HP3 & $190^{[1]}$ & $164^{[2]}$ & $372^{[3]}$ \\
vided $_{[32]}^{[3]}$ Viscosity measured at $400^{\circ} \mathrm{C}$ according to ISO 11443, values of frequency not pro- \\
\end{tabular}


- Experimentally assessed the rate of neck growth of PEK HP3 powder grains.

- Analytically determined the impact of viscoelasticity on the neck growth rate.

- VoF simulations are performed to investigate the shape effects on sintering.

- It is found that the shape effects can lead to separation of sintering units. 


\section{Declaration of interests}

$\bigotimes$ The authors declare that they have no known competing financial interests or personal relationships that could have appeared to influence the work reported in this paper.

$\square$ The authors declare the following financial interests/personal relationships which may be considered as potential competing interests:

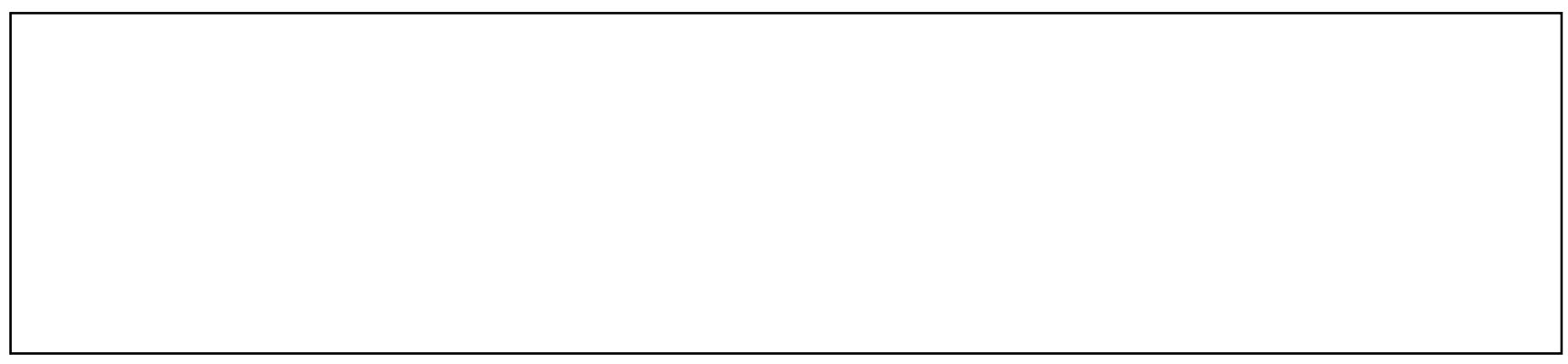


LaTeX Source Files
Click here to download LaTeX Source Files: LatexSourceFiles.zip

LaTeX Source Files
Click here to download LaTeX Source Files: LatexSourceFiles.zip Click here to download LaTeX Source Files: LatexsourceFiles.zip

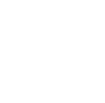

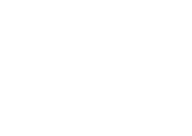
$\sqrt{2}$

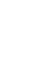

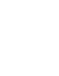

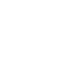
(1) (1) (1) (1)

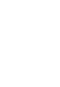
(1) .

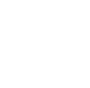
更

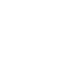

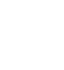

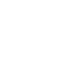

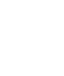

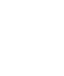

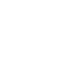

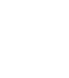

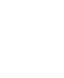

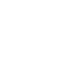

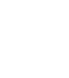

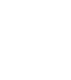

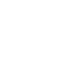

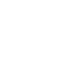

